\title{
Editorials
}

\section{What can we do to reduce the burden of avoidable deaths in those with serious mental illness?}

\author{
DAVID CROMPTON, AARON GROVES, JOHN MCGRATH
}

\begin{abstract}
Individuals with schizophrenia have higher mortality rates compared to the general community. Apart from an increased risk of suicide, people with schizophrenia have an increased risk of death related to a wide range of comorbid physical conditions. There is evidence to suggest that much of this mortality is avoidable. The provision of assertive management of comorbid physical disorders has the potential to help close the differential mortality gap. While the primary data are robust, there is less empirical evidence to guide policy makers and service providers when dealing with these problems. Focused clinical programs aimed at reducing risk factors (e.g. smoking, obesity) and shared care between mental health teams and primary care providers can help reduce the burden of avoidable deaths. In light of recent evidence suggesting that the mortality gap has widened in recent decades, there is an urgent need to address the burden of avoidable deaths in those with serious mental illnesses.

Declaration of Interest: None.
\end{abstract}

The evidence clearly shows that people with schizophrenia have an increased risk of dying from a wide range of conditions compared to the general population. The critical question now is what can we do to reduce the burden of avoidable deaths in schizophrenia? Do we need more research to guide service development or do we already know what needs to be done? If so, how can we translate the evidence into practice in an efficient and effective fashion?

There is an abundance of high-quality evidence that examines mortality in schizophrenia. While there are many different metrics related to mortality, the most widely used measure is the Standardised Mortality Ratio (SMR), which compares mortality in individuals with schizophrenia versus the general population. SMRs are calculated by dividing the 'observed' mortality in a given population (e.g. the number of deaths in a group of individuals with schizophrenia) by the 'expected' mortality in that same group as predicted by age- and sex-specific for a standard population. Thus, an SMR of 2.0 would indi-

Address for correspondence: Professor J. McGrath, Queensland Centre for Mental Health Research, The Park Centre for Mental Health, Wacol, QLD 4076 (Australia).

E-mail: john_mcgrath@qcmhr.uq.edu.au cate that individuals with schizophrenia are twice as likely to die compared to the general population during a given period. SMRs can be calculated for overall mortality ('all-cause'), or for more specific, widely-used categories (e.g. cancer, cardiovascular, endocrine, suicide etc).

A recent systematic review and meta-analysis identified 36 unique studies drawn from 19 different countries (Saha et al., 2007). Overall, the review found that while suicide contributes to the increased mortality associated with schizophrenia, individuals with schizophrenia have increased mortality risks due to a wide range of comorbid somatic conditions.

For persons, the median SMR for 'natural' causes of death was 2.41, however the estimates varied over a wide range $(10-90 \%$ quantiles $=0.99$ to 4.10$)$. Elevated SMRs were found in all of the specific causes of death apart from cerebrovascular diseases.

Unexpectedly, the differential mortality gap associated with schizophrenia has been increasing (Saha et al., 2007). The data suggested that the differential mortality gap has widened over time because the patient group had not shared in the improved health of the general community.

In recent years, evidence has accumulated showing that people with schizophrenia are less likely to receive the medical or surgical interventions commonly received by the general community. For example, research from 
Western Australia (Coghlan et al., 2001; Lawrence et al., 2003) has shown that while there was little difference in hospital admission rates for ischaemic heart disease between psychiatric patients versus the general community, psychiatric patients were less likely to receive surgical interventions (such as revascularisation procedures). In other words, those with serious mental illnesses were not receiving equitable levels of health care. Research from Canada (Kisely et al., 2007; 2008) and Italy (Amaddeo et al., 2007) has confirmed these findings.

With respect to the factors contributing to the higher SMR in schizophrenia, there are many factors that mediate pathways and barriers to health care in general (e.g. availability of services, stigma, disease profiles) (Murray \& Lopez, 1994). The onset of a psychotic disorder can result in a cascade of unhealthy lifestyle factors (e.g. poor diet, substance abuse) elevating the risk for various somatic diseases, and consequently an increased risk of death. It is thought that individuals with schizophrenia are less inclined to seek health care, consume less medical care, engage in high-risk behaviours, and are less compliant with their treatments (Coghlan et al., 2001; Hennekens et al., 2005; Mitchell \& Malone, 2006). A systematic approach to monitoring and treating the physical health needs of people with schizophrenia across the entire age spectrum is clearly warranted (Marder et al., 2004; Amaddeo et al., 2007).

This need for awareness and monitoring is also relevant to pregnant woman with schizophrenia who are at risk of adverse health events, as are their offspring (Bennedsen $e t$ al., 2001; Hauck et al., 2008).

In response to the growing awareness of avertable morbidity and deaths in those with serious mental illness, a variety of programs have been developed that seek to integrate quality primary and mental health care (Fagiolini \& Goracci, 2009; Mitchell, 2009). Primary care, mental health care providers and government have recognized the need to shift from a system where care was provided in silos, to one where the relationship between physical and mental health is seamlessly integrated.

The situation in Australian has highlighted the need for government to facilitate new linkages and work in collaboration with primary care and mental health services. A report commissioned by the Western Australian Government has explored ways to respond to comorbid physical health problems in those with serious mental illness (Office of Mental Health, 2004). The key recommendations of the report include the following:

- the need to ensure that attention to the physical health status of mental health consumers is incorporated into routine clinical protocols and properly documented;
- the need to improve the proper transfer of that information between health sectors;

- the need to promulgate the central role of general practitioners in the care and monitoring of the physical health of mental health consumers;

- the need for better training of health professionals in the area of the physical wellbeing of those affected by mental illness;

- the need for health promotion and disease prevention information to be effectively targeted to mental health consumers and their families and carers.

Further details related to this project can be found at the following website: http://www.healthright.org.au/.

Despite the development of integrated approaches to provide mental and physical health care for those with serious mental illness, significant impediments remain for this population. For example, homelessness impacts upon the capacity of care providers to engage those with serious mental illness with the general health care systems. Funding models can accentuate the difficulties of coordination across governments and health care providers with respect to integration of primary care and mental health care service. For example, the Mental Health Nursing Incentive Program introduced by the Commonwealth Government of Australian has had varying level of success, despite embedding staff within the general practice setting.

Maximising the benefits of integration will continue to require a focus on ensuring collaboration between government Mental Health Services and primary care providers. Funding needs to support the linkage between these groups. It also needs to ensure the engagement of mental health patients in a setting which, in Australia, is challenged by a continued reduction of primary care health practitioners who are willing to accept the government rebate (rather than charge the patients an additional fee on top of the rebate). This particular barrier to care further compounds the difficulty of ensuring not just access to care but also engagement with high quality evidence based care for those with serious mental illnesses.

Young people (Quine et al., 2003; Booth et al., 2004), culturally and linguistically diverse populations and Aboriginal and Torres Strait Islanders with serious mental illnesses in Australia are specific populations within this group that are further disadvantaged (Harris \& Telfer, 2001; Harris \& Zwar, 2005; Ghent, 2008; Hale-Robertson \& Scott, 2008; Thomson et al., 2008). These three groups are among the most disadvantaged of those with serious mental illness experiencing difficulty in engagement not only with the Mental Health Service but also with prima-

Epidemiologia e Psichiatria Sociale, 19, 1, 2010 
ry health care. Special services may need to be developed to target the needs of these particular groups.

While clinical practice guidelines exist in relation to medical illnesses and mental health illnesses, there appears to be a gap between the two "worlds". Audits of mental health records indicate that services frequently fail to ensure regular evaluation of their mental health patient's physical health or identify the primary care providers. Even the monitoring of metabolic factors related to the use of second generation anti-psychotic medications is at times over-looked. The training program for doctors and nurses tends to minimise the importance of physical health care in relation to mental health patients.

The development of 'super-clinics' affords the opportunity to promote the development of "one stop shops" that increase the opportunity/capacity to promote access to, early intervention with respect to mental and physical health care, while also enabling engagement with specialized medical services. It is evident that in jurisdictions where there is an amalgamation/collaboration of key agencies, there is improvement in intersectorial collaboration, which in turn increases the likelihood of improved outcomes.

The proposed $4^{\text {th }}$ National Mental Health Plan for Australia has focused on a number of aspects that seek to promote the engagement of those with serious mental illness with mental health services and general health care providers. There is a focus on well-being and recovery with supported employment and vocational programmes which ultimately will improve the socio-economic status of individuals and therefore enact as an enabler to access to general medical care. There is an aim to promote the delivery of care through integrated care centres that will provide coordinated care and improved access to a range of services.

While there is sufficient data for us to appreciate the extent of the problem, there is a lack of data that can guide us on what type of response is needed now. For example, government-funded programs in Australia have attached mental health nurses to General Practice networks. These staff actively monitor the chronic health needs of the clients with serious mental illness and provide structured intervention programs. However, while the benefits of this type of program seem self-evident, is it the best value of the health dollar? Are there other ways to incentivize patients and their families to take a more active role in their health care? How can we change the practices of mental health staff and other health providers to ensure that patients with serious mental illness get optimal physical care (Killackey et al., 2008; Sederer, 2009)? Because health systems vary widely between nations, there will not be a 'one size fits all' program. Each health service will need to develop creative ways to energize intersectorial collaboration. In summary, there is need for more service evaluation and health services research. Evaluation and research that focuses on illness and interventions is important, but it is not sufficient to guarantee improved outcomes for those with chronic illness or disability. Those with chronic illness and disability, including the subgroup with SMI, have been recognized has having multiple disadvantages which are reflected in health, justice, educational, employment and socio-economic outcomes (Coumarelos \& Wei, 2009).

These negative or social exclusion outcomes reflect the opposite of the goals of the 4th National Mental Health Plan, which has a priority to develop social inclusion for mental health care. While social exclusion continues, those with SMI will remain vulnerable with a lack of access to the social, health, educational and employment opportunities that the majority of society can access. To achieve change in outcomes necessitates a shift from social exclusion to social inclusion. In order to the address these broad issues, funding at all levels of government will be required.

It is paradoxical that as we become better at the early detection of people with schizophrenia, the relative risk of mortality in this group is worsening. Given the potential for an even greater disease burden secondary to the introduction of second-generation antipsychotic medications (Allison \& Casey, 2001; Fontaine et al., 2001), research aimed at optimizing the physical health of people with schizophrenia needs to be undertaken with a sense of urgency.

\section{REFERENCES}

Allison D.B. \& Casey D.E. (2001). Antipsychotic-induced weight gain: a review of the literature. Journal of Clinical Psychiatry 62, Suppl. 7, 22-31.

Amaddeo F., Barbui C., Perini G., Biggeri A. \& Tansella M. (2007). Avoidable mortality of psychiatric patients in an area with a community-based system of mental health care. Acta Psychiatrica Scandinavica 115, 320-325.

Bennedsen B.E., Mortensen P.B., Olesen A.V. \& Henriksen T.B. (2001). Congenital malformations, stillbirths, and infant deaths among children of women with schizophrenia. Archives of General Psychiatry 58, 674-679.

Booth M.L., Bernard D., Quine S., Kang M.S., Usherwood T., Alperstein G. \& Bennett D.L. (2004). Access to health care among Australian adolescents young people's perspectives and their sociodemographic distribution. Journal of Adolescent Health 34, 97-103.

Coghlan R., Lawrence D., Holman C.D.J. \& Jablensky A.V. (2001). Duty to Care: Physical Illness in People with Mental Illness. The University of Western Australia: Perth. 
Coumarelos C. \& Wei Z. (2009). The Legal Needs of People with Different Types of Chronic Illness or Disability. Law and Justice Foundation: Sidney.

Fagiolini A. \&, Goracci A. (2009). The effects of undertreated chronic medical illnesses in patients with severe mental disorders. Journal of Clinical Psychiatry 70, Suppl. 3, 22-29.

Fontaine K.R., Heo M., Harrigan E.P., Shear C.L., Lakshminarayanan M., Casey D.E. \& Allison D.B. (2001). Estimating the consequences of anti-psychotic induced weight gain on health and mortality rate. Psychiatry Research 101, 277-288.

Ghent A. (2008). Overcoming migrants' barriers to health. Bulletin of the World Health Organization 86, 583-584.

Hale-Robertson K. \& Scott K.S. (2008). Improving the physical health of people with SMI. Synergy 3, 11 .

Harris M.F. \& Telfer B.L. (2001). The health needs of asylum seekers living in the community. Medical Journal of Australia 175, 589-592.

Harris M. \& Zwar N. (2005). Refugee health. Australian Family Physician 34, 825-829.

Hauck Y., Rock D., Jackiewicz T. \& Jablensky A. (2008). Healthy babies for mothers with serious mental illness: a case management framework for mental health clinicians. International Journal of Mental Health Nursing 17, 383-391.

Hennekens C.H., Hennekens A.R., Hollar D. \& Casey D.E. (2005). Schizophrenia and increased risks of cardiovascular disease. American Heart Journal 150, 1115-1121.

Killackey E., Jorm A., Alvarez-Jimenez M., McCann T.V., Hides L. \& Couineau A.L. (2008). Do we do what we know works, and if not why not? Australian and New Zealand Journal of Psychiatry 42, 439-444.

Kisely S., Smith M., Lawrence D., Cox M., Campbell L.A. \& Maaten S. (2007). Inequitable access for mentally ill patients to some medically necessary procedures. Canadian Medical Association Journal $176,779-784$

Kisely S., Sadek J., MacKenzie A., Lawrence D. \& Campbell L.A.
(2008). Excess cancer mortality in psychiatric patients. Canadian Journal of Psychiatry 53, 753-761.

Lawrence D.M., Holman C.D., Jablensky A.V. \& Hobbs M.S. (2003). Death rate from ischaemic heart disease in Western Australian psychiatric patients 1980-1998. British Journal of Psychiatry 182, 31-36.

Marder S.R., Essock S.M., Miller A.L., Buchanan R.W., Casey D.E., Davis J.M., Kane J.M., Lieberman J.A., Schooler N.R., Covell N., Stroup S., Weissman E.M., Wirshing D.A., Hall C.S., Pogach L., PiSunyer X., Bigger J.T., Jr., Friedman A., Kleinberg D., Yevich S.J., Davis B. \& Shon S. (2004). Physical health monitoring of patients with schizophrenia. American Journal of Psychiatry 161, 13341349.

Mitchell A.J. \& Malone D. (2006). Physical health and schizophrenia. Current Opinion in Psychiatry 19, 432-437.

Mitchell P. (2009). Mental health care roles of non-medical primary health and social care services. Health and Social Care in the Community 17, 71-82.

Murray C.J. \& Lopez A.D. (1994). Global and regional cause-of-death patterns in 1990. Bulletin of the World Health Organization 72, 447480.

Office of Mental Health (2004). Who Is Your GP? Department of Health, Government of Western Australia: Perth.

Quine S., Bernard D., Booth M., Kang M., Usherwood T., Alperstein G. $\&$ Bennett D. (2003). Health and access issues among Australian adolescents: a rural-urban comparison. Rural Remote Health 3, 245.

Saha S., Chant D. \& McGrath J. (2007). A systematic review of mortality in schizophrenia: is the differential mortality gap worsening over time? Archives of General Psychiatry 64, 1123-1131.

Sederer L.I. (2009). Science to practice: making what we know what we actually do. Schizophrenia Bulletin 35, 714-718.

Thomson N., Burns J., Hardy A., Krom I., Stumpers S. \& Urquhart B. (2008). Overview of Australian Indigenous Health Status October 2008. Retrieved December 10, 2009, from http://www.healthinfonet.ecu.edu.au/ouroverview. 